tensicherheit von Cloud-Angeboten beurteilen, vergleichen und nachprüfen können. Mehr Informationen zum Projekt finden sich im Internet unter www.verimetrix.de .

Im Rahmen des Projekts entwickeln Partner aus Wissenschaft, Recht und Wirtschaft neue Werkzeuge, mit denen Unternehmen und Behörden Datenschutzanforderungen in Cloud-Umgebungen standardisiert beschreiben und automatisch messen können. Die im Projekt entwickelten Konzepte und Techniken bilden die Grundlage für zertifizierte und regelkonforme Cloud-Dienste, welche Datenschutz- und andere Vorgaben nachweisbar berücksichtigen. VeriMetrix betrachtet den gesamten Lebenszyklus von Cloud-Anwendungen: Von der Beschreibung und Festlegung der Datenschutzanforderungen über die Auswahl eines geeigneten Cloud-Diensteanbieters nach bestimmten Kriterien, die Überprüfung der Datenschutzbestimmungen im laufenden Betrieb bis hin zum überprüfbaren Löschen der Daten nach Ende der Nutzung.

Im Rahmen von VeriMetrix formuliert das Fraunhofer-Institut für Sichere Informationstechnologie SIT Datenschutzanforderungen für das Cloud-Umfeld, speziell für Schulen und Banken. Für diese Bereiche bestehen besondere Anforderungen und Vorschriften wie das Kreditwesengesetz oder das hessische Schulgesetz. Um die Datenschutzeigenschaften unterschiedlicher Cloud-Dienste miteinander vergleichbar zu machen, erstellen die Wissenschaftler des Fraunhofer SIT aus den spezifischen Anforderungen an den Datenschutz einen Katalog von Kenn- und Messzahlen. Darüber hinaus legen sie fest, wie die definierten Kennzahlen technisch gemessen werden können. Außerdem stellen die Wissenschaftler sicher, dass das Projekt selbst datenschutzkonform arbeitet.

Das VeriMetrix-Konsortium besteht aus den Partnern avocado rechtsanwälte, careon $\mathrm{GmbH}$, Colt Technology Services $\mathrm{GmbH}$, der Universität Münster sowie dem Fraunhofer-Institut für Sichere Informationstechnologie SIT als Konsortialführer. Das Projekt „VeriMetrix - Definition und Verifikation von Kennzahlen für den Datenschutz in Cloud-Anwendungen" ist zum 1. März dieses Jahres mit einer Laufzeit von zwei Jahren gestartet. Das Gesamtvolumen beträgt 2,7 Mio. VeriMetrix wird durch das Bundesministerium für Bildung und Forschung (BMBF) gefördert.

\section{BSI-Werkzeug LARS: Einstieg in IT-Sicherheit für Betreiber von Industriesteuerungs-Anlagen}

Das Bundesamt für Sicherheit in der Informationstechnik (BSI) stellte zur Hannover Messe interessierten Anwendern kostenfrei ein Werkzeug zur Sicherheitsanalyse und Bewertung von industriellen Steuerungsumgebungen im Rahmen einer Erprobungsphase zur Verfügung. „LARS“ (Light And Right Security) wurde im Auftrage des BSI von der Sirrix AG security technologies in Zusammenarbeit mit der TÜV SÜD Rail GmbH entwickelt.

Das Werkzeug unterstützt Betreiber von industriellen Steuerungsumgebungen (ICS, Industrial Control System) diese sicher zu implementieren und zu betreiben. Es beinhaltet Funktionalitäten, um vorhandene ICS-Umgebungen und den Umsetzungsgrad von Sicherheits-Maßnahmen in einer vereinfachten Form zu erfassen. Vergleichbar mit gängigen Compliance-Werkzeugen, bewertet LARS den aktuellen Sicherheits-Level dann aufgrund einer für die Zielgruppe optimierten Metrik.

Durch die vielfältigen Berichtsfunktionen können anschließend die Benutzer den aktuellen Status abrufen und beispielsweise er- forderliche Maßnahmen überblicken, die zur Erreichung des nächst besseren Sicherheits-Levels umgesetzt werden müssen. Umgesetzte Maßnahmen können im Tool nachgezogen werden und deren Auswirkungen auf den Sicherheits-Level werden durch das Tool neu ermittelt. Ein Mapping auf das ICS Security Kompendium des BSI und Standards wie IEC 62443, ISO 27001 und Grundschutzkataloge des BSI rundet die Funktionalität ab.

Im Vergleich zu klassischen Schutzbedarfs- und Risikoanalysen ermöglicht das Tool einen vereinfachten Einstieg in die Cyber-Sicherheit. Gerade für mittelständische Unternehmen reduziert dies sowohl die erforderlichen Erfahrungen bei Security- und Analysetechniken als auch den Personalaufwand. Das Tool wird für kleine und mittelständische Unternehmen mit bis zu 1.000 Mitarbeitern empfohlen und ist als erster Einstieg gedacht.

Interessierte Anwender können das Werkzeug direkt beim BSI anfordern.

\section{DuD 2014: 16. Jahresfachkonferenz 23. und 24. Juni 2014 in Berlin}

- Die EU-Datenschutzgrundverordnung und ihre Auswirkungen

- Das IT-Sicherheitsgesetz

- Mobile Geräte und Anwendungen (Apps) und der Datenschutz

- Die NSA-Überwachungsaffäre und ihre Folgen

- Beschäftigtendatenschutz in der Praxis

- Google-Brille

- Aktuelles zum Thema Videoüberwachung

" Podiumsdiskussion: „Wieviel Staat braucht die IT-Sicherheit?“

- und viele andere Themen

Am 23. und 24. Juni 2014 treffen sich zum 16. Mal Datenschutzbeauftragte, Anwälte und IT-Sicherheitsverantwortliche zum Gedanken- und Erfahrungsaustausch in Berlin.

Die DuD-Konferenz ist bekannt für ihre professionelle und kommunikative Atmosphäre. Das Get Together am Vorabend der Konferenz sowie großzügige Konferenzpausen und ein festliches Dinner am Abend des ersten Konferenztages tragen zu einem entspannten Ambiente bei und animieren zum vertieften, fachlichen Gedankenaustausch in außergewöhnlichem Rahmen.

Weitere Informationen und Anmeldung: $h t t p: / / w w w . c o m p u t a s$. de/konferenzen/dud_2014/DuD_2014.html

\section{ARTIKEL 29 Gruppe der EU genehmigt Microsofts Cloud-Verpflichtungen}

This Working Party was set up under Article 29 of Directive 95/46/ EC. It is an independent European advisory body on data protection and privacy. That is the official writing of the committee at Microsoft: Brussels, 2 April 2014

Ms Dorothee Belz

Associate General Counsel

Legal and Corporate Affairs

Microsoft EMEA

By email: Dorothee.Belz@Microsoft.com

Dear Ms Dorothee Belz,

The EU Data Protection Authorities have analyzed the reply of Microsoft (email sent by Jean Gonié on 6th February 2014) relating to a 